\title{
Abnormal cervical cancer screening results in OBGYN clinics: differences in follow-up
}

\author{
Frances W Grimstad ${ }^{1 *}$, Catherine L Satterwhite ${ }^{2}$, Jennifer Schuchmann ${ }^{1}$, Elaina Murray ${ }^{3}$, Katie Brophy ${ }^{3}$ and Kevin Ault ${ }^{1}$ \\ ${ }^{1}$ Department of Obstetrics and Gynecology, University of Kansas, Kansas City, KS, USA \\ ${ }^{2}$ Department of Preventative Medicine and Public Health, University of Kansas, Kansas City, KS, USA \\ ${ }^{3}$ School of Medicine, University of Kansas, Kansas City, KS, USA
}

\begin{abstract}
Context: Cervical cancer screening is the primary tool for cervical cancer detection, and prevention. There are minimal safety nets in place at our institution to track screenings that are delinquent in nature.

Aims: Evaluate abnormal cervical cancer screening follow-up in an outpatient Obstetrics and Gynecology (OBGYN) clinic to determine factors associated with recidivism rates.

Methods: A retrospective analysis of the abnormal cervical cancer screening follow-up of patients between postgraduate-staffed OBGYN clinics, and faculty-staffed OBGYN clinics in an outpatient facility setting was conducted. Evaluation included all cervical cancer screenings conducted on women aged $\geq 21$ years during a twoyear academic period. To determine differences by provider type, chi-square tests, and t-tests were conducted.

Results: Of the 4,158 cervical cancer screenings performed, $17.6 \%$ were abnormal. Patients in postgraduate clinics were younger than in faculty clinics (34 vs 38 years, $P<0.0001)$, and were less likely to return for follow-up $(62.5 \%$ vs $79.7 \%, p<0.0001)$. More abnormal test results were ordered, and reviewed by the same person in attending clinics than postgraduate clinics $(90.1 \%$ vs $43.1 \%, P<0.001)$. Among patients with an abnormal result, $53.0 \%$ in faculty clinics were established patients, compared to $24.3 \%$ of postgraduate clinic patients $(P<0.0001)$.

Conclusions: Irrespective of provider type (postgraduate or faculty), established patients are more likely to follow up after an abnormal cervical cancer screening than first-time patients. Endeavors will be put towards creating a tool for first-time patients to help improve awareness of the importance, and the process of, cervical cancer screenings in our clinics.
\end{abstract}

\section{Introduction}

Cervical cancer screening is the primary tool for cervical cancer detection, and prevention. Screenings at our health system are regularly performed in our Obstetrics and Gynecology department. There are minimal safety nets in place to track cervical cancer screenings that are delinquent in nature.

We strive to ensure the highest compliance with cervical cancer screening protocols, yet we lack data regarding which patients are most at risk for being lost to follow-up. The study goal was to identify patients least likely to follow-up, and aim to develop a targeted recall system to reduce our institutions cervical cancer screening recidivism rate.

\section{Methods}

Our objective was to evaluate abnormal cervical cancer screening follow-up in outpatient Obstetrics, and Gynecology (OBGYN) clinics to determine factors associated with recidivism rates. To evaluate this objective, we performed a retrospective chart review, of all patients with cervical cancer screenings performed at our health system's ObGyn clinics by postgraduates, and faculty providers who were practicing for the entire two-year period during the 2013-2015 clinical years. We did this to ensure that continuity of care over the course of the year was possible with each provider, as well as to capture a time frame in which our Electronic Medical Record (EMR) was in use. Our null hypothesis was that postgraduate clinic patients were less likely to return for care compared to faculty clinic patients.

We included all cervical cancer screenings performed by these providers on patients over the age of 21 . Women were excluded for any of the following: their initial screening was not performed at our health system's laboratory (these were patients who had screenings performed at outside laboratories, and presented to our clinic for review of results), or was performed inpatient, in the high-risk obstetrics clinic, the urogynecology clinic, the reproductive endocrinology, and infertility clinic, or the gynecologic oncology clinic.

Data was grouped by provider type: postgraduate versus faculty clinic. Basic demographics, patient characteristics, abnormal screening results, and follow-up information were collected.

Correspondence to: Frances W Grimstad, Pediatric and Adolescent Gynecology Fellow, Children's Mercy Hospital/ University of Missouri Kansas City, USA, Tel: 816404 1000,E-mail: grimstadf@umkc.edu

Key words: cervical cancer screening, recidivism, recall rates, continuity clinics, postgraduate training

Received: September 23, 2017; Accepted: October 23, 2017; Published: October 25, 2017 
To determine differences by provider type, chi-square tests, and t-tests were conducted.

\section{Results}

Overall, 4,158 cervical cancer screenings were performed in the 2013-2015 clinical years. Of these, $17.6 \%$ were abnormal. A breakdown of the cervical cancer screening results which met initial criteria showed 3,428 of them were normal, so we did not proceed with further evaluation. Seven hundred and thirty, or $17.6 \%$ were abnormal; we screened these further (Figure 1). Of the abnormal cervical screenings, roughly three quarters, or 561, were resulted from faculty clinics and one-quarter, or 144, were from postgraduate clinics.

Due to 2013 being in the early adaptation period of our EMR, there was found to be limited to no clinic documentation on some patients. Because of this, there were $3.4 \%$ or 25 abnormal results which could not be linked to a specific type of provider. In the faculty clinic, $79.7 \%$ returned for follow-up, $16.0 \%$ did not and $4.3 \%$ were unknown due to charting ambiguity in the EMR. In the postgraduate clinics, $62.5 \%$ returned for follow-up, $32.0 \%$ did not, and $5.5 \%$ were unknown due to charting ambiguity in the EMR.

Table 1 shows some basic demographics regarding the patients who had abnormal pap smears. Of note, postgraduate patients tended to be younger, and were more likely to be a new patient to the provider. More abnormal test results were ordered and reviewed by the same person in attending clinics than postgraduate clinics ( $90.1 \%$ vs $43.1 \%$, $P<0.001)$. There were no differences in the rates between those who were new to the OB Gyn clinics, new to the institution as a whole, or rates of patients with histories of abnormal cervical cancer screenings.

Figure 2 shows the percent of patients who return for follow-up in a clinic juxtaposed against the percent of patients who are preestablished for this clinic. Faculty clinic has a higher percentage of preestablished patients as well as a higher follow-up rate.

Within the cohort of abnormal pap smears for all participants, those who were new to the provider were less likely to follow-up compared to those who were established (only $74.8 \%$ of new patients returned for follow-up compared to $85.6 \%$ of established patients $P<0.001$ ). This held true for patients who were new to the obstetrics, and gynecology clinic as a whole with abnormal pap smears as well (only $74.5 \%$ of new patients to the clinic compared to $83.7 \%$ of established patients $P=0.003)$. When stratified by attending, and postgraduate clinics this held true for attending patients but not postgraduate patients.

When compared to faculty clinic patients, those in postgraduate clinics were less likely to follow-up after an abnormal screening $(79.7 \%$

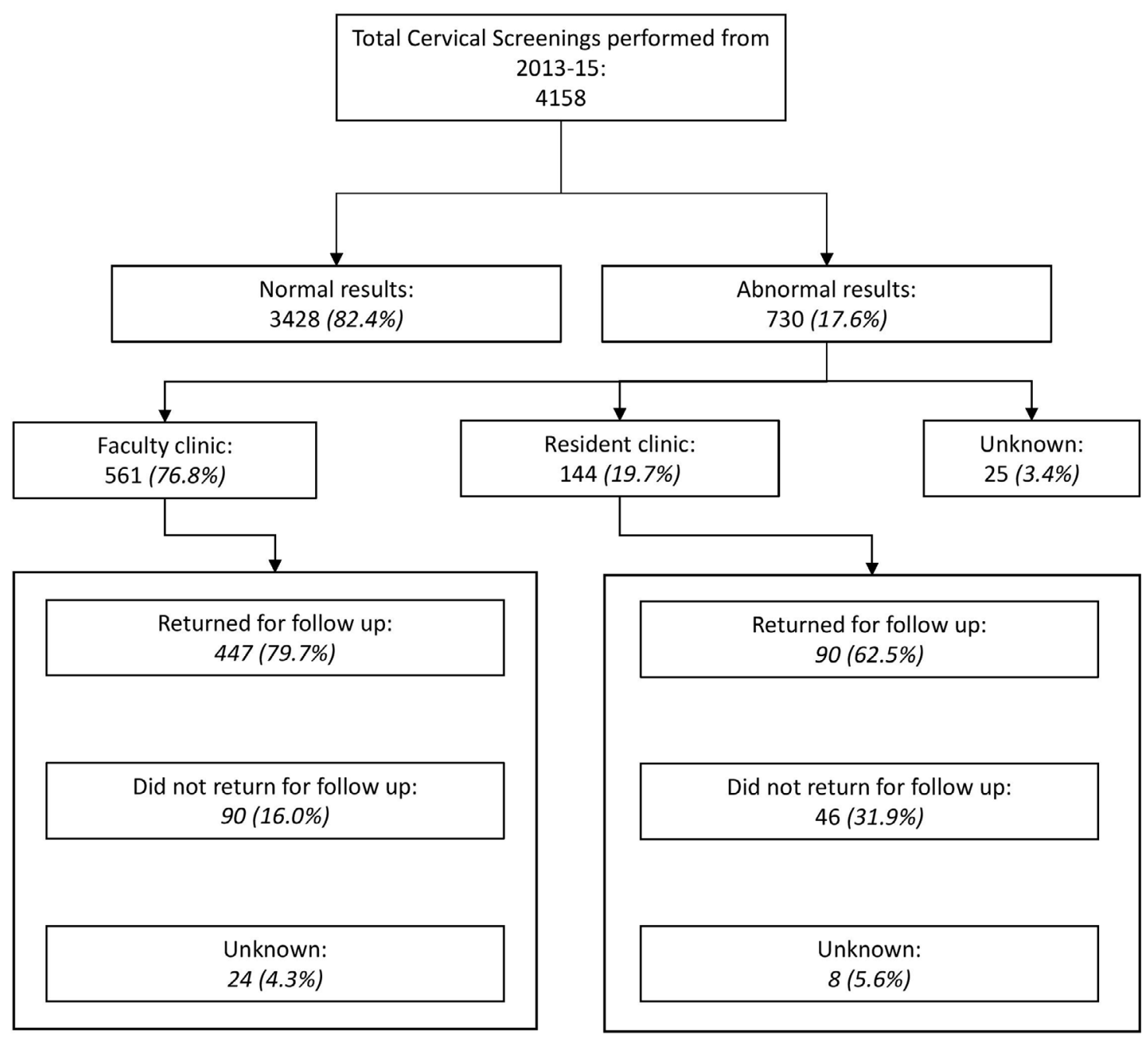

Figure 1. Breakdown of cervical cancer screenings performed in the clinic from 2013-2015 


\section{Comparison of patients who returned for follow up and patients who were preestablished by clinic type}

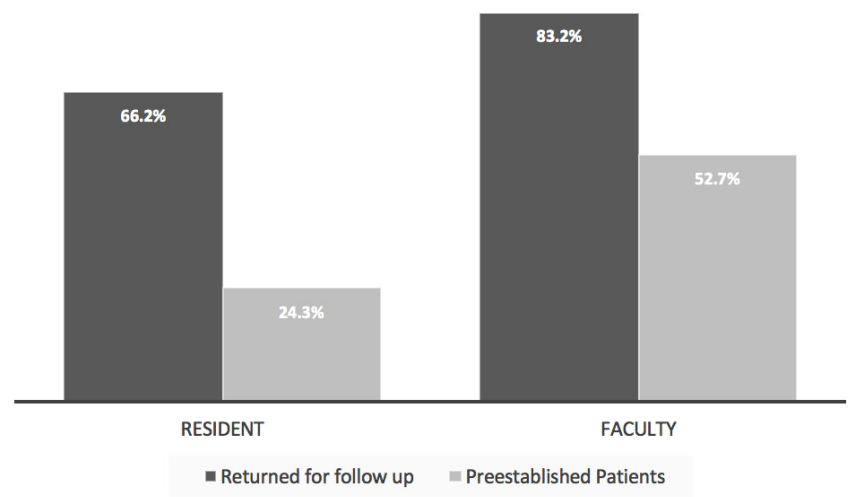

Figure 2. Juxtaposition of the percent of patients who followed up after abnormal cervical cancer screenings in each clinic type compared to the percent of patients in each clinic type who were pre-established.

Table 1. Select characteristics of abnormal cervical screenings in our clinic from 20132015 stratified by provider type.

\begin{tabular}{|l|l|l|}
\hline Provider Type & Resident & Faculty \\
\hline $\begin{array}{l}\text { Number of abnormal } \\
\text { screenings (n) }\end{array}$ & 144 & 561 \\
\hline $\begin{array}{l}\text { Patient's average age (year } \\
\pm \text { SD) }\end{array}$ & $33.6 \pm 11.4$ & $37.7 \pm 13.6$ \\
\hline $\begin{array}{l}\text { New patient to this orovider } \\
\text { (\%)* }\end{array}$ & $75.70 \%$ & $47.10 \%$ \\
\hline $\begin{array}{l}\text { New patient to KUMC } \\
\text { ObGyn clinic (\%) }\end{array}$ & $48.60 \%$ & $39.60 \%$ \\
\hline New patient to KUMC (\%) & $16.70 \%$ & $6.80 \%$ \\
\hline $\begin{array}{l}\text { Patients with a history of } \\
\text { abnormal screenings (\%) }\end{array}$ & $50.70 \%$ & $52.40 \%$ \\
\hline $\begin{array}{l}\text { Patients whose result was } \\
\text { reviewed by the same person } \\
\text { who ordered the test * }\end{array}$ & $43.1 \%$ & $90.70 \%$ \\
\hline
\end{tabular}

$* p<0.001$; KUMC: University of Kansas Medical Center

in the postgraduate clinic compared to $62.5 \%$ in faculty clinics $P<0.001$ ) which was a statistically significant finding. Faculty clinic patients were also twice as likely to be established (52.9\% versus $24.3 \%)$ which was also a statistically significant finding.

\section{Discussion}

Cervical cancer is still a prevalent disease for women. There American Cancer Society estimated approximately 12,000 new cases of cervical cancer in 2015 in the United States. ${ }^{1}$ When the cervical cancer screening was invented in the 1920s by Georgios Papanikolaou, and Aurel Babes it was a turning point in the field of early diagnosis, and treatment of cervical pathology. The mortality rate has decreased by more than $50 \%$ secondary to screening techniques but was still quoted at 2.3 per 100,000 women in 2011 in the United States (USA) [1]. However, these rates are purportedly underestimated. New literature supports that data which excludes patients who have undergone hysterectomies (and thus only includes those with cervixes and are still at risk) shows the rates of 10.1 per 100,000 black women and 4.7 per 100,000 white women in the USA [2].

The American Society for Colposcopy, and Cervical Pathology (ASCCP) came out with the latest guidelines in 2012 that have described expectations for the screening of cervical cancer, and the management of abnormal results [3]. However, in 2016 the American College of Obstetrics and Gynecology (ACOG) published a bulletin noting that approximately $60 \%$ of cervical cancer diagnosis are a result of inadequate screening [1]. A large meta-analysis of studies which reviewed screening patterns performed on patients who ultimately were diagnosed with cervical cancer found that $11.9 \%$ of them failed to follow up after an abnormal screening was resulted [4]. Improvements in these screening programs are difficult in training settings where continuity of care may be an issue.

Historically our clinic has followed the model which most USA clinics use, which has been to rely upon patients to schedule the follow up; but research has shown that this is not an effective model. One large Swedish study evaluated the success of various recall methods including invitation letters, reminders, and phone calls. They noted that a combination of the above methods improved follow-up compared to any method in isolation, especially when all three were employed. This suggests that there is a benefit to creating multiple layers of reminders for the patients in our clinic [5]. Another study noted that having blanket reminder systems for all cohorts would sometimes have negative effects on those who did not require it, thus suggesting that targeting reminders to those who needed it most could have a better benefit [6].

In an effort to improve outcomes at other sites, multiple studies have evaluated various call, and recall models to improve screening compliance including phone calls, letters and automated reminders in EMR. While no one method has proven superior, we look to these for guidance on how we might approach our own clinic [6-8]. We recognize that as in many clinics the ability to change scheduling templates can be difficult to overcome, and having alternative resources to capture patients who are likely to fall through the cracks are needed. Given that the new patients in our study cohort appear to be most at risk of failure to follow up we look to targeted improvements aimed at that particular population.

Strengths of this report were the large cohort, thorough documentation for a majority of patients and a diverse patient body. Weaknesses of this report include its retrospective set up, the dilution of provider specific information due to the sheer number of providers present during the study period, and the inability to garner a large sample as the years prior did not take advantage of electronic medical records. Another weakness is the lack of data on demographics, and follow-up outcomes of patients with normal pap smears. Due to a large number of pap smears performed during the study time period, this was deemed infeasible for our study team. Given this, we were not able to compare overall traits of patients within the clinics we studied but only demographics, and comparative outcomes within the abnormal population.

Overall, this study looked to decipher explicit groups within our diverse patient body to target improvement in their recall rates. We did find that postgraduate clinic patients had higher recidivism rates, but that this held true more for new patients than return patients, even those who were treated by multiple providers. While other clinics may have different demographics of patients who fail to return for followup it is important to note that our clinic is fairly diverse, and serves a wide array of patients across private faculty clinics and postgraduate run clinics. Across all of these demographics, we found that patients who were presenting for the first time were more likely to fail to return for follow up of abnormal pap smears, suggesting that a diverse array of clinics may benefit from this information as we included both private faculty patients as well as postgraduate clinic patients. 


\section{Conclusion}

In conclusion, our clinic data suggests that established patients are more likely to follow up after an abnormal cervical cancer screening than first-time patients, and that this is seen irrespective of the type of provider (postgraduate or faculty). Targeting first-time patients is thus seen as an important step in reducing recidivism in the clinic. Future endeavors will be put towards creating a tool for first-time patients to help improve awareness of the importance, and process of, cervical cancer screenings in our ObGyns clinic.

\section{Acknowledgement}

We would like to acknowledge Erin Hecker MD and Dani Cothern MD who contributed to the design process of this report.

\section{References}

1. Practice Bulletin No. 168 Summary (2016) Cervical Cancer Screening and Prevention. Obstet Gynecol 128: 923.
2. Beavis AL, Gravitt PE, Rositch AF (2017) Hysterectomy-corrected cervical cancer mortality rates reveal a larger racial disparity in the United States. Cancer 123: 10441050. [Crossref]

3. Massad LS, Einstein MH, Huh WK, Katki HA, Kinney WK, et al. (2013) 2012 updated consensus guidelines for the management of abnormal cervical cancer screening tests and cancer precursors. Obstet Gynecol 121: 829.

4. Spence AR, Goggin P, Franco EL (2007) Process of care failures in invasive cervical cancer: systematic review and meta-analysis. Prev Med 45: 93 [Crossref]

5. Eaker S, Adami HO, Granath F, Wilander E, Sparen P (2004) A large population-based randomized controlled trial to increase attendance at screening for cervical cancer. Cancer Epidemiol Biomarkers Prev 13: 346-354. [Crossref]

6. Burack RC, Gimotty PA, George J, McBride S, Moncrease A, et al. (1998) How reminders given to patients and physicians affected pap smear use in a health maintenance organization: results of a randomized controlled trial. Cancer 82: 23912400. [Crossref]

7. Stein K, Lewendon G, Jenkins R, Davis C (2005) Improving uptake of cervical cancer screening in women with prolonged history of non-attendance for screening: a randomized trial of enhanced invitation methods. J Med Screen 12: 185-189. [Crossref]

8. Rashid, R. M., Ramli, S., John, J. et al. (2014) Cost effective analysis of recall methods for cervical cancer screening in Selangor--results from a prospective randomized controlled trial. Asian Pac J Cancer Prev 15: 5143-5147. [Crossref]

Copyright: (C2017 Grimstad FW. This is an open-access article distributed under the terms of the Creative Commons Attribution License, which permits unrestricted use, distribution, and reproduction in any medium, provided the original author and source are credited. 